\title{
Measuring sit-to-stand timing variability over time using under mattress pressure sensor technology
}

\author{
Theresa Grant ${ }^{1,2}$, Vilas Joshi ${ }^{3}$, Member, IEEE, Matthew Taylor ${ }^{3}$, Member, IEEE, Frank Knoefel ${ }^{1,3}$, Heidi Sveistrup $^{2}$, Martin \\ Bilodeau $^{1,2}$, Jeffrey Jutai ${ }^{1,4}$ \\ 1. Bruyère Research Institute. Bruyère Continuing Care. Ottawa, Canada \\ 2. School of Rehabilitation Sciences. University of Ottawa. Ottawa, Canada \\ 3. Department of Systems and Computer Engineering. Carleton University. Ottawa, Canada \\ 4. Interdisciplinary School of Health Sciences. University of Ottawa, Canada
}

\begin{abstract}
Getting out of bed is a fundamental activity of daily living and one that supports independent living. Under mattress pressure sensor technology represents a way to monitor changes in this basic but critical mobility task among older adults at risk of institutionalization. However, little is known about normal variations in this ability over time in the home context. This study used under mattress pressure sensors to measure and analyze the variability of sit-to-stand (STS) timing in a community-dwelling older adult. A pressure-sensitive mat was installed in the participant's home and left in place to collect information over a period of nine months. A processing algorithm was developed to extract the STS phase of the first morning bed exit from which STS time could be measured. STS timing data were visualized using a histogram and analyzed for trends over the extended period using nonparametric regression and wavelet analysis. Results indicate that the analytical methods used were able to identify trends in STS timing as well as highlight deviations. The ability to collect and analyze the variability of STS timing using this pressure sensitive technology combined with the analysis methodology provides clinicians with a way to assess mobility remotely in the home setting.
\end{abstract}

Keywords - pressure sensors; patient monitoring; bed exit; sit-to-stand; variability; home context

\section{INTRODUCTION}

The ability to get out of bed is a simple and regularly performed activity of daily living. As individuals age, changes can occur that make this task more difficult and threaten the ability of older adults to live independently. In particular, rising from the sitting position requires sufficient strength and stability which often become compromised due to conditions associated with aging [1][2][3]. Early detection and intervention for declining mobility can extend the independence of older adults and allow them to remain in their own homes for as long as possible. The sit-to-stand task is of particular interest to geriatric researchers since it is an indicator of general physical function [2][3]. Sit-to-stand (STS) timing is the most commonly studied dimension of this task [2] and has been shown to differentiate older adults who fall and those who do not [1].

A number of technologies have been used to measure STS timing including wearable accelerometers; gyroscopes and video $[4][5][6]$. Under mattress pressure sensor technology presents a non-invasive method for measuring STS timing. It has the advantage of being less intrusive than video and does not require that individuals wear or carry a devices such as an accelerometer or gyroscope. Under mattress pressure sensor technology has been used in controlled laboratory environments [7][8][9] and can differentiate between healthy older adults and those with mobility impairments on the basis of STS time [10]. It follows, therefore, that installing this type of technology in the homes of older adults opens new opportunities for detecting changes in basic mobility activities that could serve as early warning systems for mobility decline. The data could also be used by clinicians to potentially track changes in bed mobility in response to health interventions such as medication changes.

In developing home monitoring systems, however, it is important to consider how the measurement of common activities like getting out of bed in the morning may vary over time in the home context and how this variability can be analyzed for clinical interpretation. Although cross-sectional STS timing values and standard deviations have been reported for different population groups and ages [2][3], no studies address the measurement of STS longitudinally as of yet.

This paper presents the use of under mattress pressure technology to measure bed exit timing in a stable community dwelling older adult over time in the home context. The study focused on a particular phase of getting out of bed- namely rising from the sitting position (sit-to-stand) since this phase of the transfer is more likely to challenge the independence of older adults due to strength and stability requirements [2]. Data were collected as part of a broader study examining the use of pressure sensor technology in monitoring bed mobility among older adults. The key contribution of this study is that it provides a methodology for analysing sit-to-stand timing variability over an extended period in the home context.

\section{DATA COLLECTION METHODOLOGY}

\section{A. Technology}

Under mattress pressure sensors manufactured by S4 Sensors Inc. were installed in the home of an 82 year old woman living independently in the community and left in place for 9 months. The pressure mat technology consists of fiber optic pressure sensors embedded in a mat capable of 
detecting pressure change. Each pressure-sensitive mat had 72 equally spaced sensors in an $8 \times 9$ grid array. The pressuresensitive mat was connected to a transmitter box which sent pressure data to a Dell OptiPlex PC via Bluetooth connection. The pressure mat and transmitter are shown in Fig. 1. The dimensions of the mat were $86 \mathrm{~cm} \times 86 \mathrm{~cm}$.

The pressure-sensitive mat was placed under the participant's mattress while the computer and transmitter box were placed under her bed. A processing algorithm was developed in MATLAB [11] to extract the STS phase of the bed exit. Each sensor in the mat was sampled at $20 \mathrm{~Hz}$. A single day of recording created approximately 500MB of data. The algorithm used down sampling techniques to quickly scan the pressure recordings and detect activity as described in a previous paper [12]. For the purpose of this study the algorithm was designed to detect the first morning exit. The first morning exit was defined as occurring between the hours of 5:00 AM and 11:00 AM, requiring the participant to have been in bed for at least 3 consecutive hours and out of bed for at least one hour. These parameters helped eliminate bathroom visits, short naps or using the bed to sit from being detected as first morning exits. All first morning exits were analyzed with the same algorithm process.

The processing algorithm relied on the percent pressure load feature. This was a summation of all the pressure sensor values over time normalized into the range of $0-100 \%$. An initial calibration was performed to find minimum and maximum pressure values as determined by loaded and unloaded conditions (i.e., bed occupancy and no bed occupancy). To measure the STS timing, three phases were distinguished; lying, sitting, and standing.

The standing phase was identified as any period when the pressure sensor was unloaded. The lying and sitting phases could be differentiated using the percent pressure load feature. Using the first data point in a standing phase, the algorithm looked backwards for the time of highest pressure which corresponded with the sitting position.

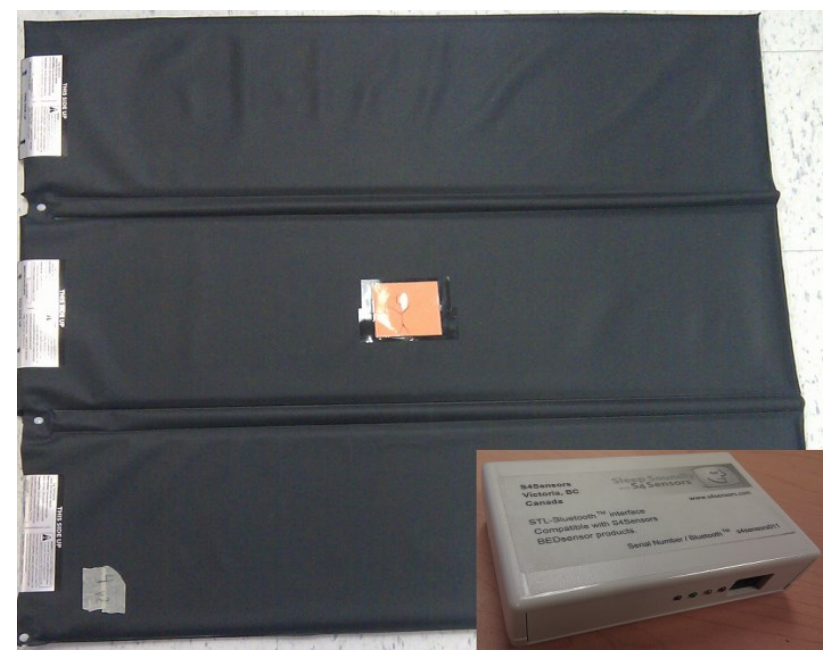

Fig. 1. Under mattress pressure sensor mat with transmitter.

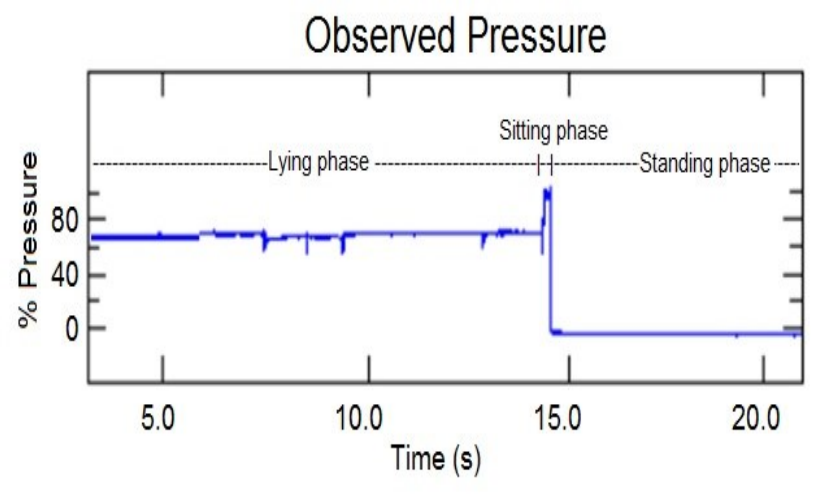

Fig. 2. Example plot of observed pressure load for a bed exit.

The peak pressure was used as the start time of the STS transfer. The difference between the time of peak pressure and the first data point in the standing phase was measured. An example plot of percent pressure load for a STS transfer is shown in Fig. 2. The occupant was initially in the lying position, transferred to the sitting position near the time of 14 seconds and then exited the bed. The mat was determined to be unloaded when the pressure load dropped to $\sim 0 \%$.

The stored data were processed offline to calculate STS timing. The processing algorithm was robust enough to remove blank periods in the measured data which may be caused by the participant being away or interruptions in data collection.

\section{B. Clinical data collection:}

As part of the study, the participant was visited monthly by a research physiotherapist who performed measures of functional mobility which included gait speed, balance and strength. She was asked about critical health events such as falls, hospital visits or any other conditions that made it more difficult for her to move.

\section{DATA ANALYSIS METHODOLOGY AND RESULTS}

The measurement of STS time was analyzed using various methods to produce a comprehensive examination of the data. These methods included descriptive statistics, a frequency histogram, nonparametric regression and Daubechies- 8 wavelet analysis. The results of each method are presented in this section. In addition, we have presented a graph of the clinical measure of gait speed over time to compare with the pattern of STS time obtained through pressure-mat data analysis.

There were some gaps in data collection due to the participant's absence from home, accidental PC power disconnection and loss of Bluetooth connectivity resulting in the valid data collection for 153 days spread over a 9 month period. Fig. 3 illustrates the number of first morning bed exits recorded each month. Table 1 provides the mean and standard deviation of STS times for the participant's first morning bed exit over the measurement period. 


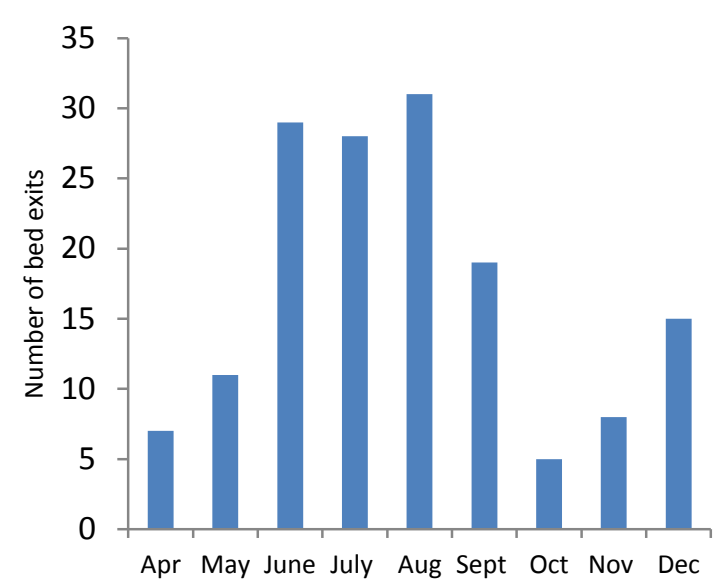

Fig. 3. Number of morning bed exits measured each month.

TABLE 1. MEAn AND StANDARD DEVIATION OF STS TIME MEASUREMENT

\begin{tabular}{|c|l|c|c|}
\hline Parameter & Mean & Standard Deviation & Number of Exits \\
\hline STS time (seonds) & 2.18 & 1.09 & 153 \\
\hline
\end{tabular}

Fig. 4 illustrates a histogram of the STS times showing the distribution of STS frequencies. These results indicate that while the majority of the STS times occurred in 2 seconds or less, there were some outliers where STS time extended to between 7 and 9 seconds.

In order to evaluate the long term trends in STS time we used a robust nonparametric regression technique (rloess) since this approach allows smoothing of the data without any need for knowledge of the shape of the curve to fit the data. A further advantage of the rloess is that it reduces the effect of outliers during regression analysis. The size used in the rloess analysis was 0.1 .

Fig. 5 provides a plot of STS time versus day along with the curve obtained using the rloess analysis. For the purpose of analysis, the calendar was compressed to illustrate the 153 days of measurements. This graph indicates that the participant's STS timing was stable over the monitored period. Specifically there was no trend towards longer times required to get out of the bed which would suggest deterioration in physical status. These results are consistent with the fact that the participant reported no critical health events and demonstrated no significant change in measures of functional mobility.

One of the mobility measures was gait speed. Fig. 6 shows the participant's gait speed over the 9 month observation period. During researcher monthly visits, the participant was instructed to walk at her comfortable walking pace over a 5 meter distance. The walk was timed and gait speed calculated in meters/second for comparison with the broader literature.

Fig. 6 illustrates that the participant's functional status in terms of gait speed did not change significantly over a period of nine months and that her gait speed was consistent with the comfortable gait speed for individuals over the age of 65 years. Gait speed for healthy individuals over the age of 65 has been reported to lie between 0.60 to $1.45 \mathrm{~m} / \mathrm{s}$ [13][14]. Although there was a slight decrease at month 4 and mild increase at month 5, values for months 7-9 stabilized again at approximately $1.2 \mathrm{~m} / \mathrm{s}$ indicating that this participant continued to walk at a speed consistent with older adults who do not have mobility impairments.

Wavelet transforms are widely used for de-noising, decompression, classification and trend analysis [15]. Wavelet transforms provide filter bank decomposition of the signal. The filter bank decomposes the signal into high frequency and low frequency bands. The low frequency signal can then be recursively subdivided into lower frequency components. This provides good resolution at low frequencies and also allows each band to be selectively investigated.

We analyzed STS timing data using wavelet transform to investigate the trends in the signal. The wavelet analysis was done using Daubechies- 8 wavelets. The decomposition was done for 4-levels.

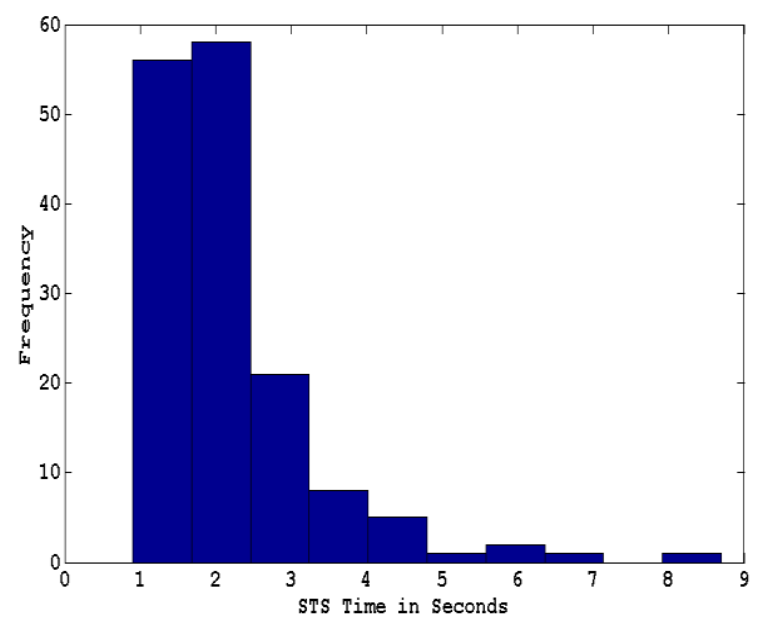

Fig. 4. Histogram of STS time in seconds.

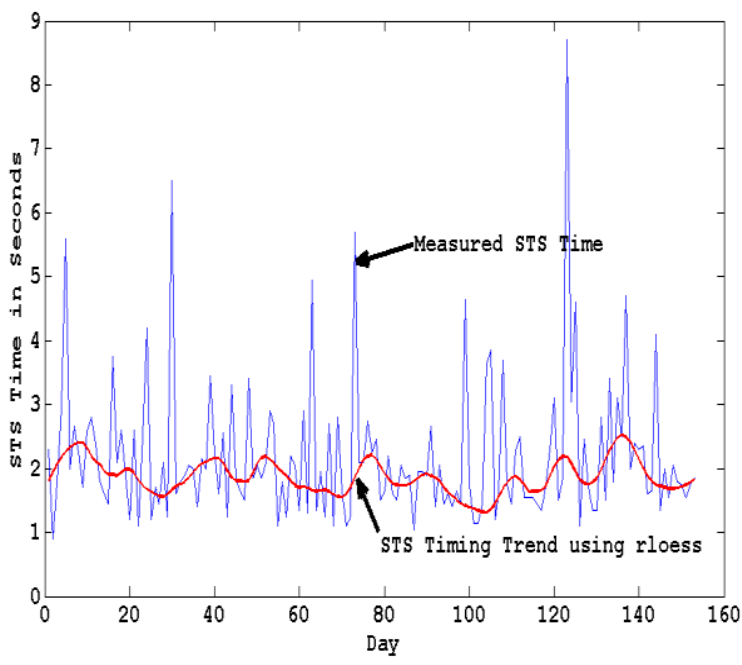

Fig. 5. Measured STS times and rloess smoothed curve versus day. 


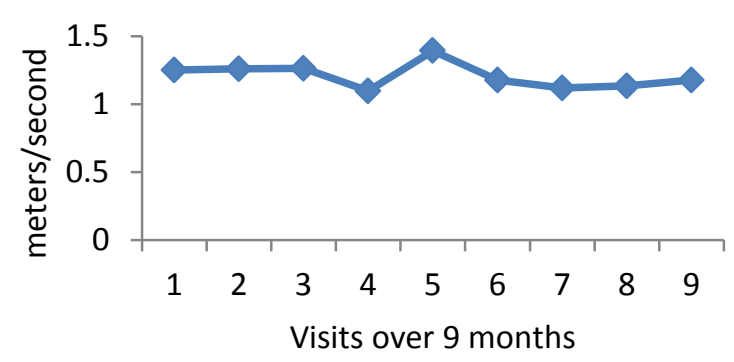

Fig. 6. Gait speed (meters/second) over 9 months.

We analyzed STS timing data using wavelet transform to investigate the trends in the signal. The wavelet analysis was done using Daubechies- 8 wavelets. The decomposition was done for 4-levels. The wavelet transform used dyadic scale (powers of 2) for decomposition. The wavelet transform coefficients were then used to reconstruct the signal at each level. Fig. 7 shows the decomposition and reconstruction of the STS timing data. Each approximation shows a trend in that particular frequency range so that we can evaluate the trend over a different time scale. The level 4 (lowest frequency component) approximation shows a trend in the lowest time scale. It is clear from the level 4 approximation that there is very little change in the participant's STS timing over the observation period. This is consistent with our analysis of the data using rloess. The advantage of wavelet analysis is that other approximations can be used to check for more short term trends.
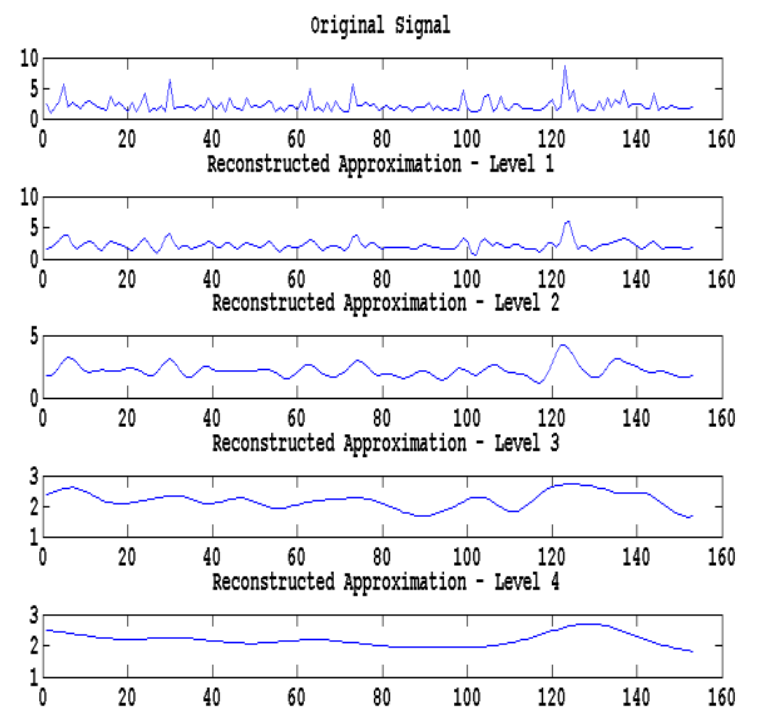

Fig. 7. Wavelet de-noising of the STS timing data.

\section{DISCUSSION}

Home monitoring of functional mobility involves the collection and processing of large amounts of data so that patterns can be identified for clinical use. The methodologies presented in this paper show how such data can be analysed to evaluate longer term trends. The results obtained indicate that bed exit STS times obtained in the home environment can be highly variable in the same healthy individual over time. Arcelus et al. [8] used similar technology in a laboratory environment to measure STS timing. These authors reported bed exit times ranging from 0.92 seconds to 1.48 seconds in a sample of 5 healthy older adults. Other laboratory studies employing alternate technologies to measure STS in healthy older adults have also reported lower levels of variability than observed in this study [16][17]. The mean bed exit STS time of $2.18(+/-1.09)$ seconds observed in our study suggests that measures obtained in the home environment may be more variable than those obtained under controlled laboratory conditions. Therefore it is necessary to employ methods for analyzing this variability which will assist in clinical interpretation.

This study found that while the majority of STS times were within 2 standard deviations of the mean, there were outliers that reached between 7 and 9 seconds. The histogram representation indicated that these times occurred infrequently in this healthy individual. Future research will need to address the extent to which the occurrence of longer STS times is indicative of mobility problems.

Nonparametric regression (rloess) and wavelet transforms of the data allowed us to examine trends over time by eliminating the effect of the outliers. The absence of a trend in either direction suggests that despite the variability observed in a raw data graph, the participant's STS bed exit time remained stable over the period of observation. The fact that this trend of stability was consistent with clinical measures over time indicates promise that bed exit measures may correspond with other dimensions of functional mobility. Gait speed was one of these clinical measures and is also a key indicator of overall health and a predictor of mortality [15]. Future research on participants with improving or declining mobility will be required to determine the extent to which trends in STS time obtained with the under-mattress technology correspond and whether the technology may assist in predicting health decline. Techniques presented in this paper provide an approach for doing so.

\section{CONCLUSION}

Under mattress sensor technology offers the ability to unobtrusively monitor bed transfer characteristics over time and thereby quantify variability in bed exit performance over the long-term. The study concludes that measures of STS bed exit time collected in a healthy individual over a 9 month period in the home context were highly variable. Results indicate that the analytical methods used were able to identify trends in STS timing as well as highlight deviations. Under mattress sensor technology shows promise for being able to assist clinicians in monitoring the functional bed mobility of older adults in the home environment.

\section{ACKNOWLEDGMENTS}

This work was funded by the Natural Sciences and Engineering Research Council (NSERC), the Canadian Institutes of Health Research (CIHR) and Carleton University. The research team thanks our study participant for 
volunteering and Melissa Donskov for her project management support.

\section{REFERENCES}

[1] E. P. Dohen et al, "An instrumented sit-to-stand test used to examine differences between older fallers and non-fallers," in Proc. IEEE Int. Conf. Engineering in Medicine and Biology Society (EMBS), Boston, Massachusetts, USA, Aug 20-Sept 3, 2011, pp. 3063-3066.

[2] W. G. M. Janssen, H. B. J. Bussmann, and H. J. Stam, "Determinants of the sit-to-stand movement: a review," Physical Therapy, vol. 82, no. 9, pp. 866-879, Sept. 2002.

[3] P. J. Millington, B. M. Myklebust, and G. M. Shambes, "Biomechanical analysis of the sit-to-stand motion in elderly persons,", 73 ed Physical Therapy Department, Zablocki VA Medical Center, Milwaukee, WI, USA, 1992, pp. 609-617.

[4] W. K. Durfee, L. Savard, and S. Weinstein, "Technical Feasibility of Teleassessments for Rehabilitation," IEEE Trans.on Neural Syst. and Rehabilitation Eng., vol. 15, no. 1, pp. 23-29, Mar. 2007.

[5] G. Hache, E. D. Lemaire, and N. Baddour, "Mobility change-of-state detection using a smartphone-based approach," in Proc. IEEE Int. Symp.on Medical Meas. and Applicat. (MeMeA), Ottawa, ON, Canada, Apr 30-May 10 2010, pp. 43-46.

[6] W. Janssen, D. G. Kulcu, H. Horemans, H. J. Stam, and J. Bussmann, "Sensitivity of Accelerometry to Assess Balance Control During Sit-toStand Movement," IEEE Trans. on Neural Syst. and Rehabilitation Eng., vol. 16, no. 5, pp. 479-484, Oct. 2008.

[7] A. Arcelus, C. L. Herry, R. A. Goubran, F. Knoefel, H. Sveistrup, and M. Bilodeau, "Determination of Sit-to-Stand Transfer Duration Using Bed and Floor Pressure Sequences," IEEE Trans. on Biomed. Eng., vol. 56, no. 10, pp. 2485-2492, Oct. 2009.

[8] A. Arcelus, I. Veledar, R. Goubran, F. Knoefel, H. Sveistrup, and M. Bilodeau, "Measurements of Sit-to-Stand timing and symmetry from bed pressure sensors," IEEE Trans. on Instrum. and Meas., , vol. 60, no. 5, pp. 1732-1740, May 2011.
[9] I. Veledar, A. Arcelus, R. Goubran, F. Knoefel, H. Sveistrup, and M. Bilodeau, "Sit-to-stand timing measurements using pressure sensitive technology," in Proc. IEEE Int.Instrum. and Meas. Technology Conference (I2MTC), Austin Texas, USA, May 2010, pp. 1337 - 1340

[10] A. Arcelus, C. L. Herry, R. A. Goubran, F. Knoefel, H. Sveistrup, and M. Bilodeau, "Determination of sit-to-stand transfer duration using bed and floor pressure sequences," IEEE Trans. on Biomed. Eng., vol. 56, no. 10, pp. 2485-2492, 2009.

[11] MATLAB version Release 2012b., Natick, Massachusetts: The MathWorks Inc., 2010.

[12] M. Taylor, T. Grant, F. Knoefel, and R. Goubran, "Bed occupancy measurements using under mattress pressure sensors for long term monitoring of community-dwelling older adults," in Proc. IEEE Int. Symp.on Medical Meas. and Applicat. (MeMeA), Gatineau, QC, Canada, May 4-5 2013, pp. 130-134.

[13] T. Steffen, T. Hacker, L. Mollinger, "Age- and gender-related test performance in community-dwelling elderly people: six-minute walk test, berg balance scale, timed up \& go test, and gait speeds.", Physical Therapy, vol. 82, no. 2, pp.128-137, Feb. 2002.

[14] S. Studenski et al, "Gait speed and survival in older adults," The Journal of the American Medical Association, vol. 305, no. 1, pp. 50-58, Jan. 2011.

[15] J. Goswami and A. Chan, Fundamental of Wavelets - Theory, Algorithms and Applications. 2nd Ed., New Jersy: Wiley, 2011.

[16] M. Goffredo, S. Schmid, M. Conforto, A. Carli, A. Neri, and T. D. Alessio, "Markerless human motion analysis in Gauss-Laguerre domain: application to sit-to-stand in young and elderly people," IEEE Trans. on Inf. Technol. Biomed., vol. 13, no. 2, pp. 209-216, Mar. 2009.

[17] B. Najafi, K. Aminian, F. Loew, Y. Leblanc, and P. Robert, "Measurement of stand-sit and sit-stand transitions using a miniature gyroscope and its application in fall risk evaluation in the elderly," IEEE Trans. on Biomed. Eng., vol. 49, no. 8, pp. 843-851, Aug. 2002. 